\title{
Prognostic Value of CEA and Ca 19.9/Tumor Markers in Gastric Cancer to Identify Patients with Poor Prognosis after Radical Surgery
}

Luigina Graziosi*, Elisabetta Marino and Annibale Donini

Department of Surgical Sciences, Radiologiche E Odontostomatologiche, University of Perugia, Italy

\begin{abstract}
Introduction: Although gastric cancer incidence has decreased worldwide, it's still the fifth most frequent malignancy and the third leading cause of cancer related mortality. Many prognostic factors have been identified as indicators of gastric cancer prognosis including tumour size, depth, lymph nodes metastasis and microvascular involvement. To date the clinical significance of tumoral markers remains unclear. In our study we would like to investigate the prognostic survival significance of preoperative CEA and CA19.9.
\end{abstract}

Material and Methods: From January 2004 to September 2016, a total of 326 gastric cancer patients were analysed. Of these 260 were enrolled in the study. The two serum tumour markers CEA and CA19.9 were detected within 7 days before surgery. The normal cut off value for serum CEA was $5 \mathrm{ng} / \mathrm{ml}$ whereas for CA19.9 was $35 \mathrm{U} /$ $\mathrm{ml}$. Patients were also dichotomized according to CEA and CA19.9 median value (respectively $2 \mathrm{ng} / \mathrm{ml}$ and $9 \mathrm{U} / \mathrm{ml}$ ).

Results: Our results suggest that the optimal application of these common tumour markers could promote the clinical screening and staging of gastric cancer patients. Their evaluation is cheap and easy, allowing a routinely use to identify patients at high risk of death or post-surgical recurrences. Also, we could utilize the optimal cut-off value of CA19.9 for individualizing patients with an early stage but a very bad prognosis.

Discussion and Conclusion: We conclude that the combined assessment of CA19.9 and CEA levels could have prognostic value in gastric cancer in particular to identify patients with a poor prognosis after radical surgery, who need an aggressive follow-up and medical treatment.

Keywords: Gastric cancer; Radical surgery; Lymph nodes metastasis; Tumour markers; Tumour size; Carcinomatosis

\section{Introduction}

Although gastric cancer incidence has decreased worldwide, it' $s$ still the fifth most frequent cancer malignancy and the third leading cause of cancer related mortality in the world [1]. Even with the advances in surgical techniques and new chemotherapy agents, the prognosis of gastric cancer remains poor due to the lack of screening system in Western Countries. During the last years many prognostic factors have been studied and identified as indicators of gastric cancer prognosis including histological subtypes, tumor size, tumor depth, HER-2 tumoral expression, lymph nodes metastasis and microvascular involvement $[2,3]$. Although many studies have also evaluated the clinical significance of serum tumoral markers; an appropriate indication for their monitoring remains unclear in gastric cancer patients. The serum levels of Carcino-embrionary antigen (CEA) and Carbohydrate Antigen 19.9 (CA19.9) may be elevated at various stage of disease and could be useful only to detect a metastatic disease with liver metastases or peritoneal carcinomatosis [4]. In fact, they demonstrated low sensitivity and specificity in particular when gastric cancer is at an early stage [5]. For this reason, the National Comprehensive Cancer Network guidelines do not recommend serum markers testing for preoperative staging and evaluation of gastric cancer patients [6]. In our study we would like to investigate the prognostic survival significance of preoperative CEA and CA19.9 and their clinical relevance to stratify patients at high risk of recurrence who could require stricter surveillance.

\section{Materials and Methods}

From January 2004 to September 2016, a total of 326 gastric cancer patients were analyzed at the Department of General and Emergency Surgery of Perugia. The inclusion criteria were as follows:
1. Diagnosis of adenocarcinoma.

2. Curative $\mathrm{D} 2$ gastric resection.

3. Preoperative evaluation of serum CEA and CA19.9.

4. Valid follow-up at the least of 6 months.

5. No others coexisting tumors.

A total of 260 gastric cancer patients were enrolled in our study. A written informed consent was obtained from all patients before surgery. The two serum tumor markers CEA and CA19.9 were detected within 7 days before surgery and measured by ELISA assay. The normal cutoff values for serum CEA and CA19.9 were respectively $5 \mathrm{ng} / \mathrm{ml}$ and $35 \mathrm{U} / \mathrm{ml}$. Preoperative data including gender, age, tumor location, histologic subtype, type of lymphadenectomy, pTNM stage, preoperative albumin, preoperative N/L, pre-operative serum CEA and serum CA19.9 were recorded as illustrated in Table 1. All patients were routinely treated with proximal, distal, or total gastrectomy associated to D2/D3 lymphadenectomy. Radical and curative surgery was defined by both the absence of any gross residual tumors from the surgical bed

*Corresponding author: Graziosi L, Marino E, Donini A, Department of Surgical Sciences, Radiologiche E Odontostomatologiche, Nuova Facoltà Di Medicina E Chirurgia Sant'Andrea Delle Fratte, 06132 Perugia, Italy, Tel: +075/5786445; Fax: +075/5786445; E-mail: luiginagraziosi@yahoo.it

Received April 25, 2018; Accepted May 14, 2018; Published May 16, 2018

Citation: Graziosi L, Marino E, Donini A, Marino E, Donini A (2018) Prognostic Value of CEA and Ca 19.9/Tumor Markers in Gastric Cancer to Identify Patients with Poor Prognosis after Radical Surgery. J Cancer Sci Ther 10: 131-134. doi: 10.4172/1948-5956.1000529

Copyright: ( 2018 Graziosi L, et al. This is an open-access article distributed under the terms of the Creative Commons Attribution License, which permits unrestricted use, distribution, and reproduction in any medium, provided the original author and source are credited. 
Citation: Graziosi L, Marino E, Donini A (2018) Prognostic Value of CEA and Ca 19.9/Tumor Markers in Gastric Cancer to Identify Patients with Poor Prognosis after Radical Surgery. J Cancer Sci Ther 10: 131-134. doi: 10.4172/1948-5956.1000529

and by pathologically negative surgical resection margin. The surgical procedure was based on the recommendations of the Japanese Gastric Cancer Treatment Guidelines [7]. The depth of primary tumor and degree of lymph node involvement were defined according to the TNM classification. Tumor size, differentiation status, tumor depth, and TNM data were also collected during the pathological examination. Patients' follow up was carried out performing enhanced chest and abdominal CT, gastroscopy and serum exams evaluation every 6 months for the first 2 years after surgery and every year for the other 7 years after surgery. The disease-free survival (DFS) was defined as the time from surgery to first disease's recurrence. The overall Survival (OS) was defined as the time from surgery either to death or from the last follow-up. All the studied patients were dichotomized both at the median and at normal value of CEA and CA19.9. The median value for serum CEA and CA19.9 were respectively $2 \mathrm{ng} / \mathrm{ml}$ and $9 \mathrm{UI} / \mathrm{ml}$. Roc curves were made in order to evaluate the specificity and sensibility of preoperative serum markers in evaluating gastric cancer $5 Y$ OS and 1-3-5Y DSF. The area under the curve was evaluated.

\section{Statistical Analyses}

Statistical analyses were conducted using PRISM software and Med Calc Software. Differences between groups were tested with a Chi-square test or T student. Survival rates were calculated using the Kaplan-Meier method, and prognostic factors and survival curves were compared using the log-rank test. Variables with a statistical P-value less than 0.05

\begin{tabular}{|c|c|c|}
\hline Features & $\begin{array}{c}\text { Absolute Number (N) } \\
\text { or Mean Value }\end{array}$ & $\%$ \\
\hline Age & 72.15 & -- \\
\hline Gender: • Male • Female & 161,99 & $61.9,38.1$ \\
\hline $\begin{array}{c}\text { Tumour location: Upper, Body, } \\
\text { Lower }\end{array}$ & $48,70,142$ & $18.5,27,54.5$ \\
\hline $\begin{array}{c}\text { Lauren classification: Intestinal, } \\
\text { diffuse }\end{array}$ & $1,27,133$ & $48.8,51.2$ \\
\hline TNM stage: I, II, III, IV & $54,49,81,76$ & $20.8,18.8,31.2,29.2$ \\
\hline Lymphadenectomy: D1, D2, D3 & $29,156,69$ & $15,60,25$ \\
\hline $\begin{array}{c}\text { Preoperative markers: Cea>5, } \\
\text { Ca1 9.9>35, double positive }\end{array}$ & $50,66,22$ & $19.1,25.6,8.5$ \\
\hline
\end{tabular}

Table 1: Patients clinical pathological features.

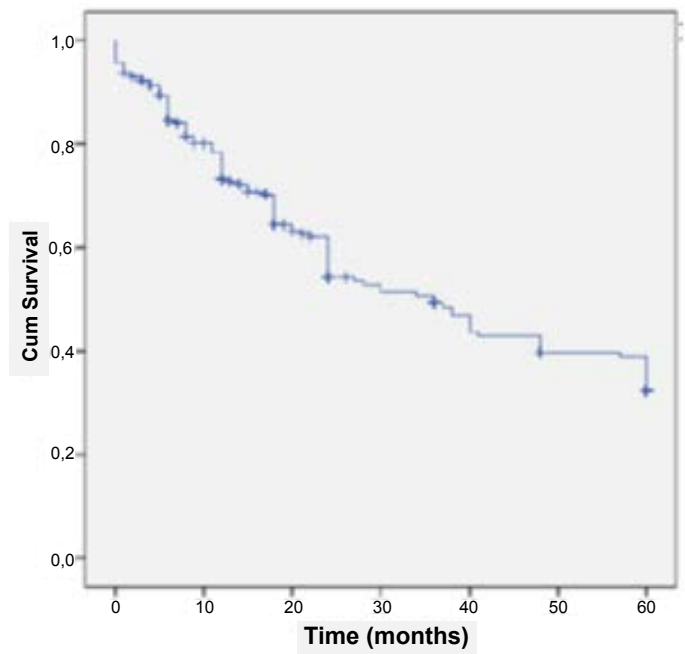

in the univariate analysis were entered into a multivariate Cox regression analysis. A P-value $<0.05$ was deemed to be statistically significant. Roc curves were also performed with the help of statistical software's.

\section{Results}

In this study 161 males (61.8\%) and 99 females (38.2\%) were included. Patients' age ranged from 37 to 94 years (median: 72 yo, mean: 72.15$)$. Gastric Cancer patients at stage I were 54 (20.8\%), at stage II were $49(18.2 \%)$ and at stage III-IV $157(60 \%)$. We performed D2 lymphadenectomy in $60 \%$ of patients; D3 in $25 \%$ and D1 in $15 \%$ of the studied population. Gastric resection with Y-en Roux reconstruction was performed in 142 patients and total gastrectomy with Y-en Roux reconstruction in 118 patients. Gastric resection was associated to other organ resection in 59 patients (22.7\%) and Hyperthermia intraperitoneal Chemotherapy (Hipec) procedure was performed in 22 patients $(8.5 \%)$. The 1-, 3- and 5-year Overall Survival (5y-OS) rates of all patients were $72 \%, 50 \%$ and $40 \%$ respectively (Figure 1). Median survival was 35 months. The 1, 3 and 5-year Disease Free Survival (1-3-5y-DFS) rates of all patients were $82 \%, 60 \%, 58 \%$ respectively (Figure 1 ). Median DFS was 42 months. $25.6 \%$ of patients resulted positive for pre-operative CA19.9 value whereas $19.1 \%$ of patients were positive for pre-operative CEA value according to the normal serum value. Only the $8.5 \%$ of patients were positive for both CA19.9 and CEA.

The distribution of CA19.9 between the categories of status (alive or death), lymph nodes positivity, local invasion was not casual with a prevalence of elevated value of CA19.9 in death population ( $\mathrm{p}=0.024)$, in lymph-node positivity $\left(\mathrm{N}^{+}\right)$population $(\mathrm{p}=0.005)$ and in advance local disease (T2/T3) population $(\mathrm{p}=0.06)$. Moreover, high levels of CA19.9 were associated with poor prognosis (5-year OS: $47 \%$ vs. $28 \%, \mathrm{P}=0.02$ and a mean survival of $37 \mathrm{vs} .29$ months Figure 2) dichotomizing patients according to the normal serum value. This positive prognostic value was lost when the patients were dichotomized considering the median value of $10 \mathrm{ng} / \mathrm{dl}$. Analyzing stage, I patients with a pre-operative value of CA19.9>10 ng/dl, a median survival of $46 v s .59$ months and a $5 \mathrm{y}-\mathrm{OS}$ of $58 \%$ vs. $90 \%$ were shown $(\mathrm{p}=0.029)$.

This result is more prominent in intestinal subtype gastric cancer patients (5-year OS: $90 \%$ vs. $40 \%, \mathrm{P}=0.04$ and a mean survival of 58 vs. 37 months (Figures 3). This prognostic importance of CA19.9 in

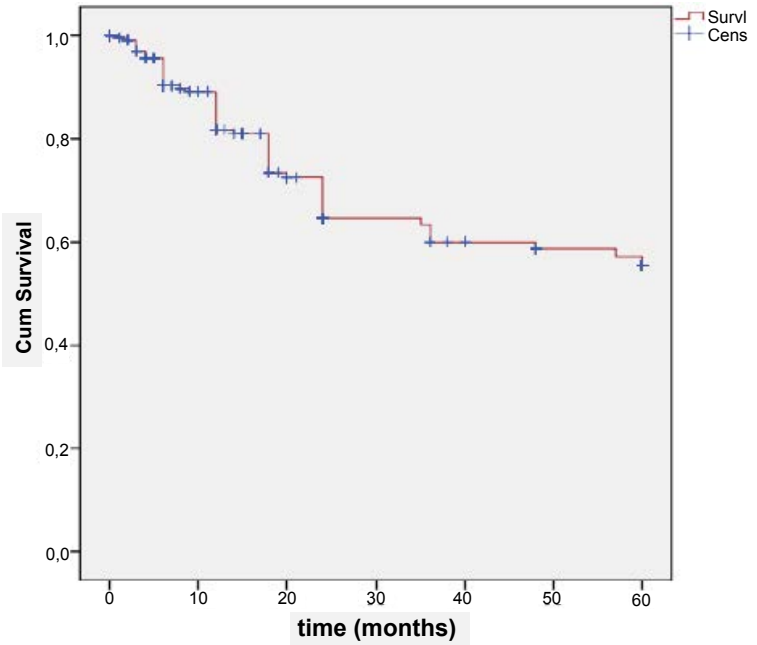

Figure 1: A. 1-, 3- and 5y-OS rates of all patients is $72 \%, 50 \%$ and $40 \%$ respectively; B. 1, 3 and 5 -y DFS) rates of all patients is $82 \%, 60 \%, 58 \%$ respectively. 
Citation: Graziosi L, Marino E, Donini A (2018) Prognostic Value of CEA and Ca 19.9/Tumor Markers in Gastric Cancer to Identify Patients with Poor Prognosis after Radical Surgery. J Cancer Sci Ther 10: 131-134. doi: 10.4172/1948-5956.1000529

earlier stages of disease was well illustrated by ROC curve (Figures 4) in which CA19.9 sensitivity and specificity for the optimal value of $12 \mathrm{ng} /$ $\mathrm{dl}$, are respectively 89 and $77 \%$, with an area under the curve of 0.874 . Moreover, high levels of CA19.9>10 ng/dl were associated with a poor 5y-DFS (5-year OS: $70 \%$ vs. $100 \%, \mathrm{P}=0.02$ in Figure 5).

There were no differences in terms of OS and DFS considering preoperative levels of CEA when analyzed with normal cutoff. On the other hand, identifying as CEA cut-off the median value, the median survival was much higher in patients with negative CEA (40 vs. 31 months survival) as the $5 \mathrm{y}$-OS (50\% vs. $38 \%$ patients $\mathrm{p}=0.03)$. CEA became a good predictor of survival in locally advanced gastric cancer; $\mathrm{pN}+$ or $\mathrm{pT} 3, \mathrm{~T} 4$ patients had higher survival rates when CEA was negative. In fact, in $\mathrm{N}^{+}$patients median survival was 34 vs. 24 months

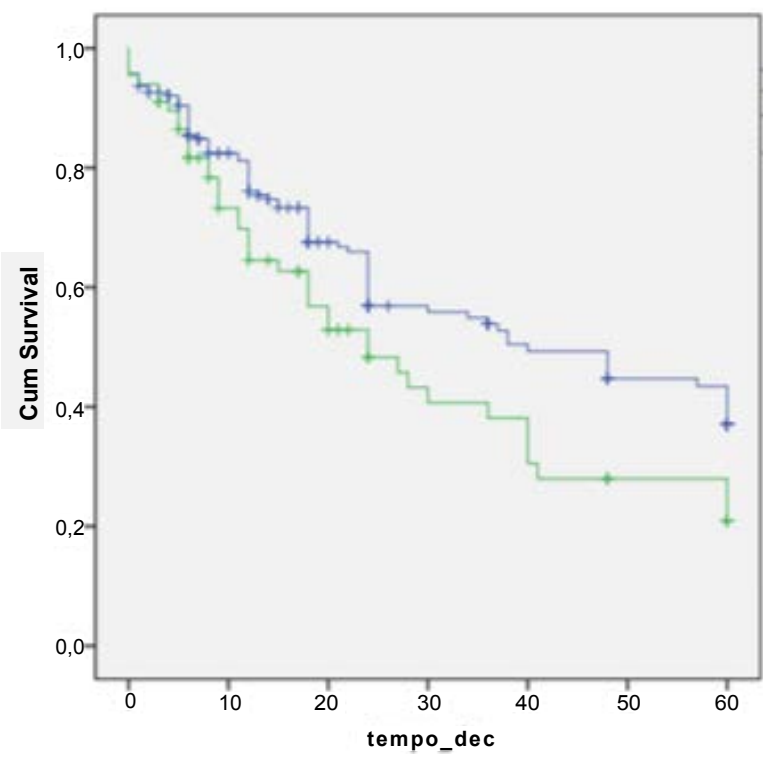

Figure 2: A 5-year OS dichotomizing patients according to the normal CA19.9 serum value: $47 \%$ vs. $28 \%, P=0.02$

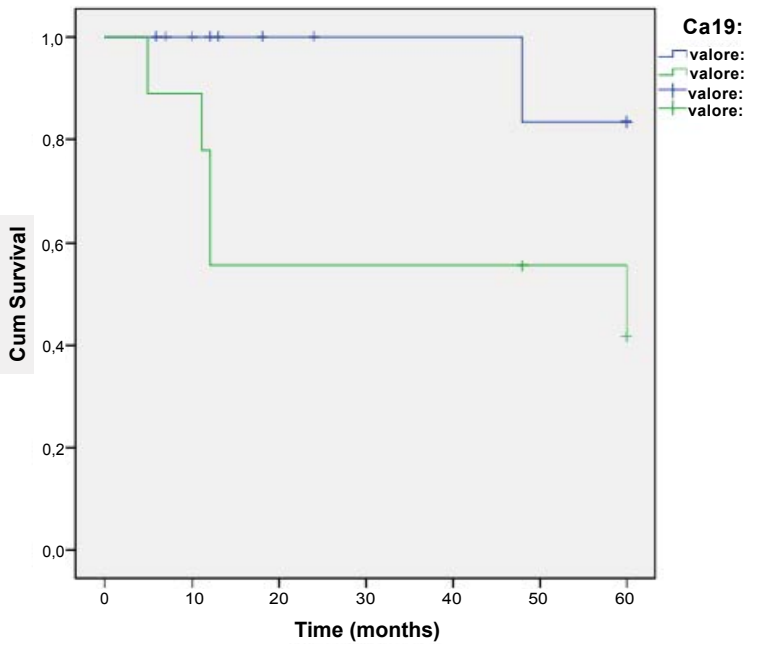

Figure 3: OS according to CA19.9 values in intestinal subtype gastric cancer patients (5-year OS: $90 \%$ vs. $40 \%, P=0.04$.

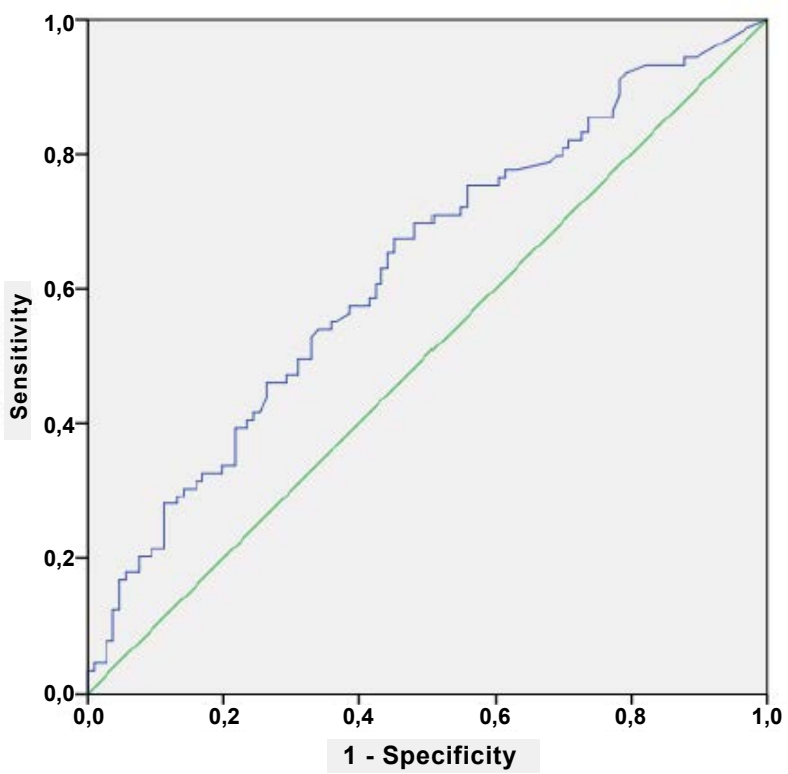

Figure 4: ROC curve forstage I, CA19.9 sensitivity and specificity are respectively $89 \%$ and $77 \%$ with an area under the curve (AUC) of 0.874

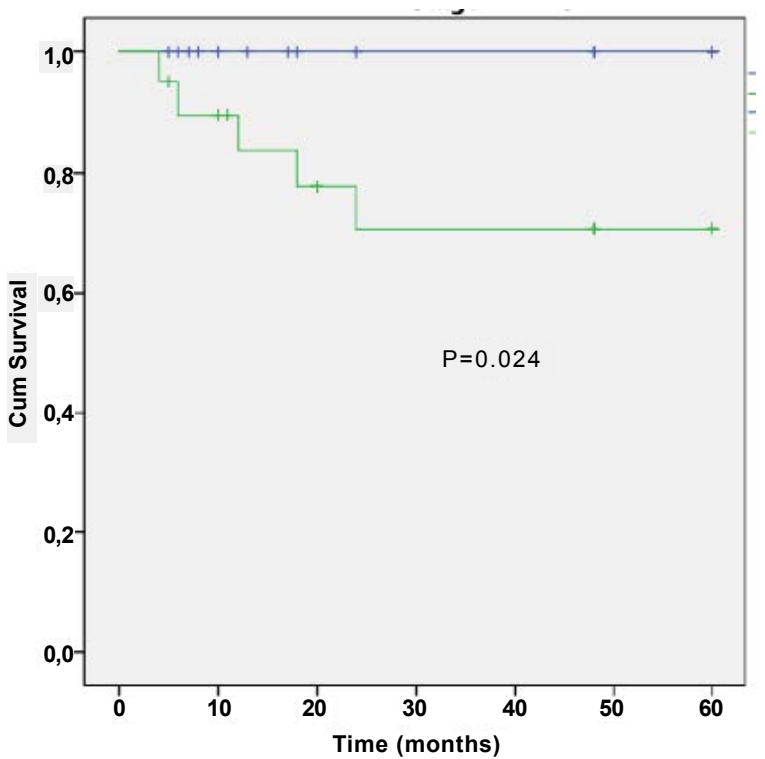

Figure 5: DSF according to CA19.9 values, 5-DSF: $70 \%$ vs. $100 \%, P=0.02$.

$(\mathrm{p}=0.03)$; in $\mathrm{T} 3 / \mathrm{T} 4$ patients median survival was 35 vs. 25 months $(\mathrm{p}=0.02)$.

\section{Discussion}

It is well known that the serum levels of various tumor markers such as CEA and CA19.9 are elevated in patients with gastric cancer; however, there are still controversies in the clinical use of these tumor markers [8]. As a matter of fact, The National Comprehensive Cancer Network guidelines do not recommend serum marker testing for preoperative evaluation and staging of gastric cancer because of their low rates of sensitivity and specificity. The Task Force of the Japanese Gastric Cancer Association recently made a systematic review that 
Citation: Graziosi L, Marino E, Donini A (2018) Prognostic Value of CEA and Ca 19.9/Tumor Markers in Gastric Cancer to Identify Patients with Poor Prognosis after Radical Surgery. J Cancer Sci Ther 10: 131-134. doi: 10.4172/1948-5956.1000529

evaluated 657 publications related to serum tumor markers in patients with gastric cancer. They concluded that monitoring tumor markers that were elevated before surgery or chemotherapy could be useful for detection of recurrence or evaluation of the therapy response [9]. Elevated preoperative serum CEA, CA19.9, AFP, and CA125 have been previously associated with a poor prognosis in gastric cancer.

A meta-analysis by Wobbes, et al., [10] of 14,651 gastric cancer patients found that elevated serum CEA was an independent prognostic risk factor of survival. Similarly, another meta-analysis of 11,408 gastric cancer patients showed that elevated serum CA19.9 was associated with a poor prognosis [11]. A strong correlation between CEA and CA19.9 levels and clinicopathological patients' features has been reported in the past by various study as in our one by Ucar, et al., [12]. We demonstrated that the clinical value was much stronger in patients with intestinal subtype adenocarcinoma. In particular the difference of survival was so exciting that CA19.9 median value could be utilized to stratify patients at the high risk of death who may benefit of chemotherapy or of a strict post-surgical follow-up. Its survival prognostic value is more evident in earlier stages when the risk of post-surgical recurrences could be potentially low. In addition, ROC curve for CA19.9 was very interesting because the optimal cut-off value was similar to our median value.

Our results seem to be in contrast to others studies suggesting that differentiation has little impact on gastric carcinoma marker level. On the other hand, CEA demonstrated to be a better predictor of recurrences in the $\mathrm{N}_{0}$ subgroup that regularly does't undergo to chemotherapy. Thus, this subgroup of patients could benefit of a post-surgical strict oncological follow-up to make an early recurrence diagnoses and to start an early therapy. There are several limitations to our study. First of all, it was a retrospective study of a single center's experience. Multi-center studies are needed to verify our findings. Secondly, the sample size was not so large compared to eastern one. Least but not last, the prognostic value of normal, CEA and CA19.9 levels for gastric cancer patients after radical gastrectomy during follow-up were not investigated.

\section{Conclusion}

Our results suggest that the optimal application of these common tumor markers could promote the clinical screening and staging of gastric cancer patients. Their evaluation is cheap and easy, allowing a routinely use to identify patients at high risk of death or post-surgical recurrences. Also, we could utilize the optimal cut-off value of CA19.9 for individualizing patients with an early stage but a very bad prognosis. The clinical utility of CEA and CA19.9 serum markers for patients with gastric cancer should be clarified in a larger multi centric study.

\section{References}

1. Jemal A, Center MM, De Santis C, Ward EM (2010) Global patterns of cancer incidence and mortality rates and trends. Cancer Epidemiol Biomarkers Prev 19: 1893-1907.

2. Kycler W, Teresiak M, Łozinski C (2006) Prognostic factors for patients with gastric cancer after surgical resection. Rep Pract Oncol Radiother 11: 235-246.

3. Sisik A, Kaya M, Bas G, Basak F, Alimoglu O (2013) CEA and CA 19-9 are still valuable markers for the prognosis of colorectal and gastric cancer patients. Asian Pac J Cancer Prev 14: 4289-4294.

4. Kochi M, Fujii M, Kanamori N, Kaiga T, Kawakami T, et al. (2000) Evaluation of serum CEA and CA19-9 levels as prognostic factors in patients with gastric cancer. Gastric Cancer 3: 177-186.

5. Kim DH, Oh SJ, Oh CA, Choi MG, Noh JH, et al. (2011) The relationships between perioperative CEA, CA 19-9, and CA 72-4 and recurrence in gastric cancer patients after curative radical gastrectomy. J Surg Oncol 104: 585-591.

6. Shimada H, Noie T, Ohashi M, Oba K, Takahashi Y (2014) Clinical significance of serum tumor markers for gastric cancer: A systematic review of literature by the Task Force of the Japanese Gastric Cancer Association. Gastric Cancer 17: 26-33.

7. Japanese Gastric Cancer Treatment Guidelines 2014 (ver. 4) (2017) Gastric Cancer 20: 1-19.

8. Deng K, Yang L, Hu B, Wu H, Zhu H, et al. (2015) The prognostic significance of pre-treatment serum CEA levels in gastric cancer: A meta-analysis including 14651 patients. Plos One 10: e124151.

9. Song YX, Huang XZ, Gao P, Sun JX, Chen XW, et al. (2015) Clinicopathologic and prognostic value of serum carbohydrate antigen 19-9 in gastric cancer: $A$ meta-analysis. Dis Makers pp: 1-11

10. Wobbes T, Thomas CM, Segers MF, Nagengast FM (1992) Evaluation of seven tumor markers (CA 50, CA 19-9, CA 19-9 TruQuant, CA 72-4, CA 195 carcinoembryonic antigen, and tissue polypeptide antigen) in the pre-treatment sera of patients with gastric carcinoma. Cancer 69: 2036-2041.

11. Park SH, Ku KB, Chung HY, Yu W (2008) Prognostic significance of serum and tissue carcinoembryonic antigen in patients with gastric adenocarcinomas. Cancer Res Treat 40: 16-21.

12. Ucar E, Semerci E, Ustun H, Yetim T, Huzmeli C, et al. (2008) Prognostic value of preoperative CEA, CA 19-9, CA 72-4, and AFP levels in gastric cancer. Adv Ther 25: 1075-1084. 